\title{
Vibrational heating of molecules adsorbed on insulating surfaces using localized photon tunneling
}

\author{
B. N. J. Persson, ${ }^{1,2}$ T. Kato, ${ }^{2}$ H. Ueba, ${ }^{2}$ and A. I. Volokitin ${ }^{1,3}$ \\ ${ }^{1}$ IFF, FZ-Jülich, 52425 Jülich, Germany \\ ${ }^{2}$ Department of Electronics, Toyama University, Gofuku, Toyama, Japan \\ ${ }^{3}$ Samara State Technical University, 443100 Samara, Russia
}

(Received 16 November 2006; revised manuscript received 13 February 2007; published 11 May 2007)

\begin{abstract}
We calculate the (average) temperature increase in an adsorbed molecule resulting from heat transfer (photon tunneling) from a tip located at a short distance (nanometers) from the adsorbate. For adsorbates on insulating substrates, the temperature increase may be so large as to induce local reactions-e.g., diffusion or desorption of the adsorbate. We conclude that photon tunneling may be used for the manipulation of adsorbed molecules or for the modification of thin adsorbed films.
\end{abstract}

DOI: 10.1103/PhysRevB.75.193404 PACS number(s): 82.37.Vb, 68.37.Ef, 68.37.Uv, 78.68. $+\mathrm{m}$

It is well known that for bodies separated by $d>>d_{T}$ $=c \hbar / k_{B} T$ (at room temperature $d_{T} \approx 10^{4} \mathrm{~nm}$ ), the radiative heat transfer between them is connected with traveling electromagnetic waves radiated by the bodies and does not depend on the separation $d$. For $d<d_{T}$ the heat transfer increases by many orders of magnitude due to the evanescent electromagnetic waves that decay exponentially into the vacuum; this is often referred to as photon tunneling. At present there are an increasing number of investigations of heat transfer due to evanescent waves in connection with scanning probe microscopy under ultrahigh vacuum conditions. $^{1-9}$

The radiative heat transfer due to the evanescent electromagnetic waves (photon tunneling) may be used for surface modification. Thus, if a hot tip is brought $\sim 1 \mathrm{~nm}$ from a surface with a thin layer of heat sensitive polymer, one may induce local polymerization and this may be used for nanoscale lithography. Such measurements with the tip in direct contact with the polymer film have already been performed. ${ }^{10}$ However, enough heat transfer for polymerization may occur also for noncontact if the tip-substrate separation is short enough and the tip temperature high enough. This noncontact mode of surface modification may have several advantages as compared to the contact mode; e.g., no wear or contamination of the tip will occur.

In this brief communication we show that a sharp tipe.g., a scanning tunneling microscope (STM) or atomic force microscope (AFM) tip — can be used for local heating of the surface, resulting in local desorption or decomposition of molecular species, and this offers further possibilities for control of local chemistry at the surface. Vibrational heating (vibrational ladder climbing) by inelastic tunneling electrons from a tip of the STM has been a great subject in single adsorbate motions. ${ }^{11,12}$

Here we study the radiative heat transfer between an adsorbed molecule on a tip and another molecule adsorbed on a substrate. We show that the temperature increase at the adsorbed molecule may be very large, which may induce local chemical reactions-e.g., diffusion or desorption. Heat transfer to some adsorbate vibrational mode-i.e., vibrational heating-will be particularly important when the energy relaxation time $\tau_{\mathrm{b}}$ of the adsorbate mode is long compared to a relaxation time characterizing the photon tunneling from the tip to the substrate adsorbate. High-frequency adsorbate vibrations on metals typically have very short energy relaxation times (in the picosecond range) owing to the continuum of low-energy electronic excitations. ${ }^{13,14}$ However, low-frequency adsorbate vibrations-e.g., frustrated translations-may have rather long relaxation times (typically of the order of nanoseconds for inert adsorbates on noble metals), ${ }^{15}$ and in these cases photon tunneling heat transfer may be important. Adsorbate vibrational modes on insulators may have very long relaxation times if the resonance frequency is above the top of the bulk phonon band. In these cases energy relaxation is caused by multiphonon processes which often are very slow. One extreme example is $\mathrm{CO}$ adsorbed on $\mathrm{NaCl}$ crystals, ${ }^{16}$ where $\tau_{\mathrm{b}} \approx 10^{-3} \mathrm{~s}$. For this case even a very weak coupling to a hot tip may result in heating of the $\mathrm{C}-\mathrm{O}$ stretch vibration.

The photon tunneling energy transfer per unit time from the tip adsorbate to the substrate adsorbate (see Fig. 1) is given by $^{8}$

$$
J=\frac{2 \hbar}{\pi} \int_{0}^{\infty} d \omega \omega \frac{\left(8 / d^{3}\right)^{2} \operatorname{Im} \alpha_{\mathrm{a}} \operatorname{Im} \alpha_{\mathrm{b}}}{\left|1-\left(8 / d^{3}\right)^{2} \alpha_{\mathrm{a}} \alpha_{\mathrm{b}}\right|^{2}}\left[n\left(\omega / T_{\mathrm{a}}\right)-n\left(\omega / T_{\mathrm{b}}\right)\right],
$$

where the Bose-Einstein factor

$$
n(\omega / T)=\frac{1}{\exp \left(\hbar \omega / k_{\mathrm{B}} T\right)-1} .
$$

We will focus on the energy transfer from a vibrational mode (frequency $\omega_{\mathrm{a}}$ and vibrational relaxation time $\tau_{\mathrm{a}}^{*}$ ) of the tip adsorbate to a vibrational mode $\left(\omega_{\mathrm{b}}, \tau_{\mathrm{b}}^{*}\right)$ of the substrate adsorbate. We also introduce the vibrational energy relaxation times $\tau_{a}$ and $\tau_{b}$. We assume that the molecular vibrations can be treated as harmonic oscillators and assume the molecular polarizabilities

$$
\alpha_{\mathrm{a}}=\frac{\alpha_{\mathrm{va}}}{1-\frac{\omega}{\omega_{\mathrm{a}}}\left(\frac{\omega}{\omega_{\mathrm{a}}}-\frac{i}{\tau_{\mathrm{a}}^{*} \omega_{\mathrm{a}}}\right)},
$$




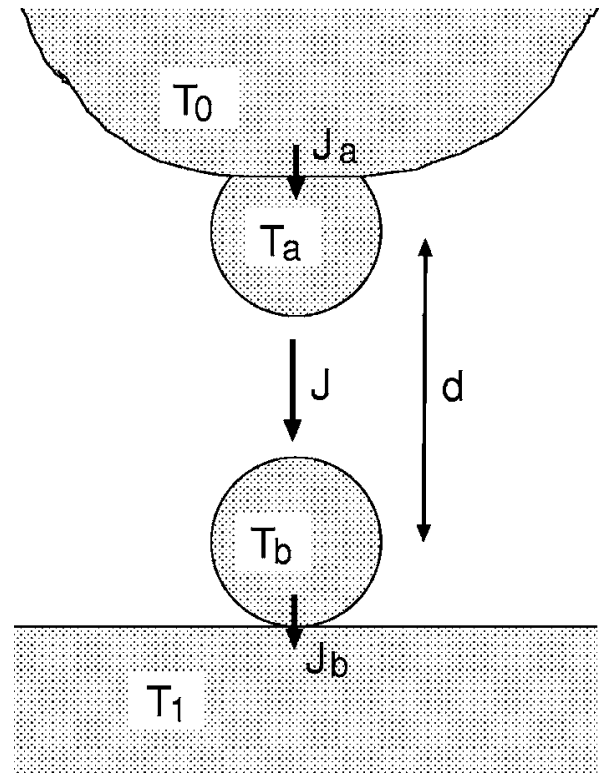

FIG. 1. The heat transfer (photon tunneling) between a tip atom (or molecule) and a substrate atom (or molecule).

$$
\alpha_{\mathrm{b}}=\frac{\alpha_{\mathrm{vb}}}{1-\frac{\omega}{\omega_{\mathrm{b}}}\left(\frac{\omega}{\omega_{\mathrm{b}}}-\frac{i}{\tau_{\mathrm{b}}^{*} \omega_{\mathrm{b}}}\right)} .
$$

Note that in general $1 / \tau_{a}^{*}>1 / \tau_{a}$ and $1 / \tau_{b}^{*}>1 / \tau_{b}$ since the vibrational relaxation rate $1 / \tau^{*}$ which enters in the polarizability has contributions from both energy relaxation and pure dephasing. ${ }^{19}$ The energy transfer rate from the tip to the tip adsorbate is given by ${ }^{17,18}$

$$
J_{\mathrm{a}}=\frac{\hbar \omega_{\mathrm{a}}}{\tau_{\mathrm{a}}}\left[n\left(\omega_{\mathrm{a}} / T_{0}\right)-n\left(\omega_{\mathrm{a}} / T_{\mathrm{a}}\right)\right],
$$

and the energy transfer rate from the substrate adsorbate to the substrate is

$$
J_{\mathrm{b}}=\frac{\hbar \omega_{\mathrm{b}}}{\tau_{\mathrm{b}}}\left[n\left(\omega_{\mathrm{b}} / T_{\mathrm{b}}\right)-n\left(\omega_{\mathrm{b}} / T_{1}\right)\right] .
$$

In general the integral (1) must be performed numerically, but as an illustration let us consider the case where the relaxation time $\tau_{\mathrm{b}}^{*}>>\tau_{\mathrm{a}}^{*}$. In this case Eq. (1) reduces to

$$
J=r \frac{\hbar \omega_{\mathrm{b}}}{\tau_{\mathrm{b}}}\left[n\left(\omega_{\mathrm{b}} / T_{\mathrm{a}}\right)-n\left(\omega_{\mathrm{b}} / T_{\mathrm{b}}\right)\right]
$$

where

$$
\begin{gathered}
r=\frac{\left(\tau_{b} / \tau_{b}^{*}\right) s}{1+\left[2\left(\omega_{\mathrm{a}}-\omega_{\mathrm{b}}\right) \tau_{\mathrm{a}}^{*}\right]^{2}+4 s}, \\
s=64 \omega_{\mathrm{a}} \tau_{\mathrm{a}}^{*} \omega_{\mathrm{b}} \tau_{\mathrm{b}}^{*} \alpha_{\mathrm{va}} \alpha_{\mathrm{vb}} / d^{6}
\end{gathered}
$$

Note that the energy transfer rate $J_{\mathrm{a}}$ (and similar for $J_{\mathrm{b}}$ ) depends only on the energy relaxation rate $1 / \tau_{\mathrm{a}}$ and not on the relaxation rate $1 / \tau_{\mathrm{a}}^{*}$ which determines the width of the vibrational resonance state. The latter is the sum of $1 / \tau_{\mathrm{a}}$ and a pure dephasing contribution which reflect the fluctuation $\omega_{\mathrm{a}}(t)$ in the vibrational level position due to the irregular thermal motion of the atoms in the system and which depend on the anharmonic coupling between the different vibrational modes. This level fluctuation contributes to the vibrational linewidth as observed using, e.g., infrared spectroscopy, but not to the energy transfer between the adsorbate and the solid on which it is adsorbed. On the other hand, the energy transfer rate $J$ between the two adsorbates is determined by the overlap in the vibrational resonance states and it is therefore determined by $\tau_{\mathrm{a}}^{*}$ (and $\tau_{\mathrm{b}}^{*}$ ), and does not depend on $\tau_{\mathrm{a}}\left(\right.$ or $\left.\tau_{\mathrm{b}}\right)$. [Note that the $\tau_{\mathrm{b}}^{-1}$ factor which appears in Eq. (6) cancels out against the factor $\tau_{\mathrm{b}}$ in the expression for $r$.]

In the high-temperature limit $n(\omega / T) \approx k_{\mathrm{B}} T / \hbar \omega$ and assuming that this relation holds for all modes and temperatures relevant here, we get

$$
J=r \tau_{\mathrm{b}}^{-1} k_{\mathrm{B}}\left(T_{\mathrm{a}}-T_{\mathrm{b}}\right) .
$$

We also get

$$
\begin{aligned}
& J_{\mathrm{a}}=\tau_{\mathrm{a}}^{-1} k_{\mathrm{B}}\left(T_{0}-T_{\mathrm{a}}\right), \\
& J_{\mathrm{b}}=\tau_{\mathrm{b}}^{-1} k_{\mathrm{B}}\left(T_{\mathrm{b}}-T_{1}\right) .
\end{aligned}
$$

Assuming first a steady-state situation so that $J=J_{\mathrm{a}}=J_{\mathrm{b}}$ we get, from Eqs. (8)-(10), $T_{\mathrm{a}} \approx T_{0}$ and

$$
T_{\mathrm{b}} \approx T_{1}+\frac{r}{1+r}\left(T_{0}-T_{1}\right)
$$

where we have assumed that $\tau_{\mathrm{b}}>>\tau_{\mathrm{a}}$.

The theory above can also be used to estimate the time it takes to reach the steady state where the (ensembleaveraged) adsorbate temperature equals (11). In general we have

$$
\hbar \omega_{\mathrm{b}} \frac{d}{d t} n\left[\omega_{\mathrm{b}} / T_{\mathrm{b}}(t)\right]=J(t)-J_{\mathrm{b}}(t) .
$$

In the classical limit this gives

$$
\frac{d T_{\mathrm{b}}}{d t}=-\frac{1}{\tau_{\mathrm{b}}}(1+r) T_{\mathrm{b}}+\frac{1}{\tau_{\mathrm{b}}}\left(T_{1}+r T_{0}\right) .
$$

If we assume $T_{\mathrm{b}}(0)=T_{1}$, this gives

$$
T_{\mathrm{b}}(t)=T_{1}+\frac{r}{1+r}\left(T_{0}-T_{1}\right)\left(1-e^{-(1+r) t / \tau_{\mathrm{b}}}\right) .
$$

Thus for $t>>\tau$, where $\tau=\tau_{\mathrm{b}} /(1+r)$, the steady-state temperature has been reached.

For adsorbates on insulating substrates $\tau_{\mathrm{b}}$ will, in general, be very large if the resonance frequency $\omega_{\mathrm{b}}$ is well above the highest substrate phonon frequency. We now consider this case, which is equivalent to low temperature. Assume for simplicity that the temperature of the substrate vanishes $\left(T_{1}\right.$ $=0)$ and assume that $\hbar \omega_{\mathrm{a}}>>k_{\mathrm{B}} T_{0}$ and $\hbar \omega_{\mathrm{b}}>>k_{\mathrm{B}} T_{0}$. In this case it is easy to show from Eqs. (4)-(6) that $T_{\mathrm{a}} \approx T_{0}$ and

$$
T_{\mathrm{b}} \approx \frac{\omega_{\mathrm{b}} T_{0}}{\omega_{\mathrm{b}}+T_{0} \ln [(1+r) / r]},
$$

where we have measured the frequency in units of $k_{\mathrm{B}} / \hbar$. 


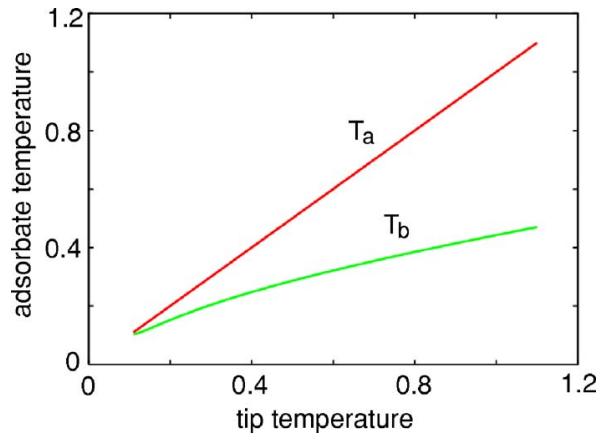

FIG. 2. (Color online) The adsorbate temperatures $T_{\mathrm{a}}$ and $T_{\mathrm{b}}$ as a function of the tip temperature $T_{0}$ (all in units of $\hbar \omega_{\mathrm{b}} / k_{\mathrm{B}}$ ) for $r$ $=0.25$ and $T_{1}=0.1 \hbar \omega_{\mathrm{b}} / k_{\mathrm{B}}$.

Let us now assume arbitrary tip and substrate temperatures but still assume $\tau_{\mathrm{b}} \gg \tau_{\mathrm{a}}$. Using Eqs. (4)-(6) we get $T_{\mathrm{a}}$ $\approx T_{0}$ and

$$
\frac{\omega_{\mathrm{b}}}{T_{\mathrm{b}}}=\ln \left[1+\frac{1+r}{r\left(e^{\omega_{\mathrm{b}} / T_{0}}-1\right)^{-1}+\left(e^{\omega_{\mathrm{b}} / T_{1}}-1\right)^{-1}}\right] .
$$

This expression reduces to (11) for high temperatures and to (12) for low temperatures. In Fig. 2 we show the effective temperature $T_{\mathrm{b}}$ as a function of the tip temperature $T_{0}$ when $T_{1}=0.1 \hbar \omega_{\mathrm{b}} / k_{\mathrm{B}}$ and $r=0.25$.

In Figs. 3 and 4 we show the temperature of the tip adsorbate, $T_{\mathrm{a}}$ (red curve), and of the substrate adsorbate, $T_{\mathrm{b}}$, as a function of the tip temperature $T_{0}$, all in units of $\hbar \omega_{\mathrm{b}} / k_{\mathrm{B}}$. These results have been obtained directly from Eqs. (1)-(5) by numerical integration. Note that the temperature of the substrate adsorbate increases monotonically as the energy relaxation time $\tau_{\mathrm{b}}$ increases, corresponding to a weaker adsorbate-substrate vibrational coupling. Figure 4 shows that, as expected, the mismatch of the vibrational frequency between the tip and substrate adsorbates reduces the heat transfer between the adsorbates.

Figure 5 shows the dependence of the tip- and substrate-

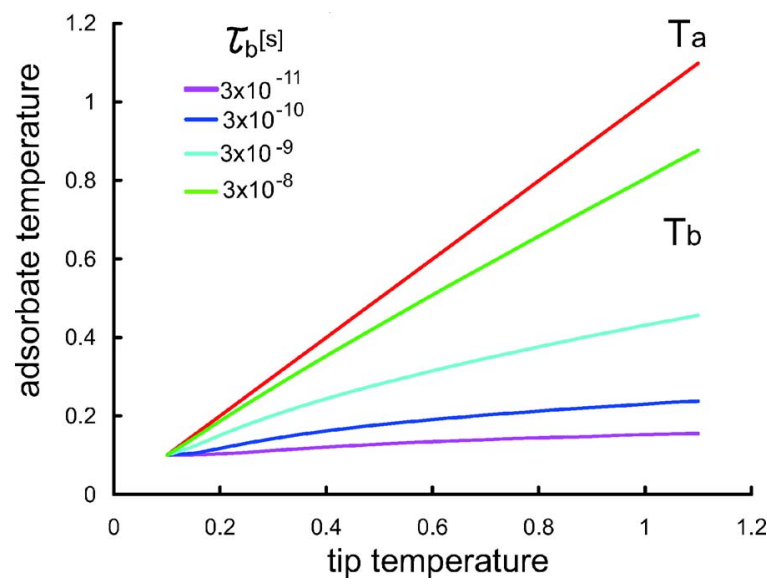

FIG. 3. (Color online) The tip- and substrate-adsorbate temperatures $T_{\mathrm{a}}$ and $T_{\mathrm{b}}$ as a function of the tip temperature $T_{0}$ (all in units of $\left.\hbar \omega_{\mathrm{b}} / k_{\mathrm{B}}\right)$. For $d=10 \AA, \quad \alpha_{\mathrm{va}}=0.2 \AA^{3}, \quad \alpha_{\mathrm{vb}}=0.04 \AA^{3}, \quad \tau_{\mathrm{a}}=3$ $\times 10^{-11} \mathrm{~s}, \tau_{\mathrm{a}}^{*}=3 \times 10^{-12} \mathrm{~s}, \tau_{\mathrm{b}}^{*}=3 \times 10^{-12} \mathrm{~s}, \omega_{\mathrm{a}}=\omega_{\mathrm{b}}=1.0 \times 10^{13} 1 / \mathrm{s}$, and $T_{1}=0.1 \hbar \omega_{\mathrm{b}} / k_{\mathrm{B}}$.

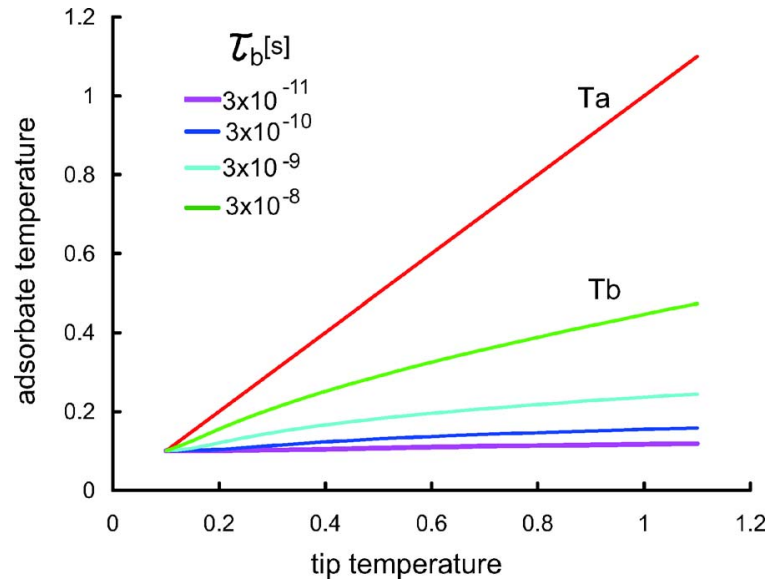

FIG. 4. (Color online) The same as Fig. 3, except for the offresonant vibrational frequency $\omega_{\mathrm{a}}=1.0 \times 10^{13} \mathrm{~s}^{-1}$ and $\omega_{\mathrm{b}}=1.1$ $\times 10^{13} \mathrm{~s}^{-1}$.

adsorbate temperatures $T_{\mathrm{a}}$ and $T_{\mathrm{b}}$ on the tip-substrate separation $d$. Note that the substrate-adsorbate temperature rapidly decreases as the separation $d$ increases.

The effective substrate-adsorbate temperature $T_{\mathrm{b}}$ calculated above may be used to calculate (or estimate) the rate $w$ of an activated process: $w=w_{0} \exp \left(-E / k_{\mathrm{B}} T_{\mathrm{b}}\right)$. When the barrier height $E$ is large, even a very small temperature increase will result in a large increase in the reaction rate. Note also that the excitation of a high-frequency mode such as the $\mathrm{C}-\mathrm{O}$ stretch mode can result in reactions-e.g., diffusion, rotation, or desorption-involving other reaction coordinates. This is possible because of the anharmonic coupling between the high-frequency mode and the reaction coordinate mode. This has already been observed in STM studies of several different adsorption systems. ${ }^{20,21}$

Let us give an example of an adsorption system where photon tunneling may give rise to a strong temperature increase. We focus on ${ }^{13} \mathrm{C}^{18} \mathrm{O}$ on $\mathrm{NaCl}(100)$ at $T_{1}=30 \mathrm{~K}$ which has been studied in detail by Chang and Ewing. ${ }^{16}$ In this case $\omega_{\mathrm{b}} \approx 2040 \mathrm{~cm}^{-1}, \tau_{\mathrm{b}} \approx 10^{-3} \mathrm{~s}$ (due mainly to decay via multiphonon emission ${ }^{16}$ ), and the (pure dephasing dominated)

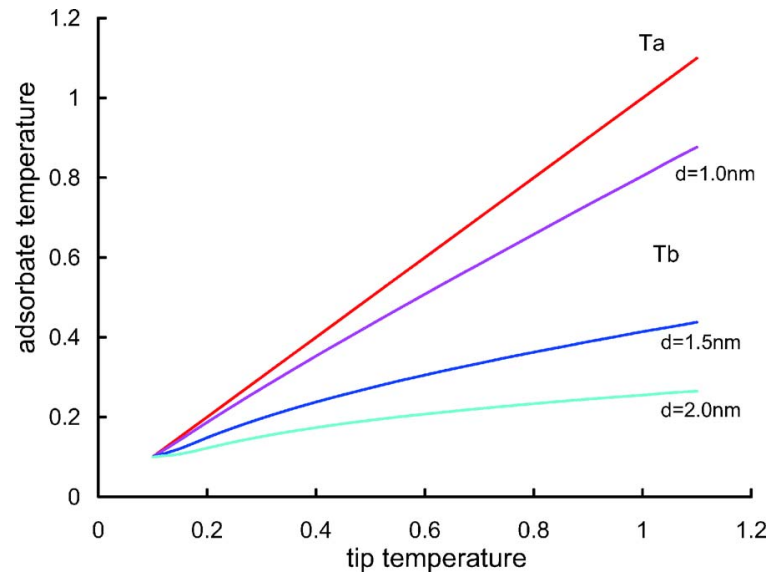

FIG. 5. (Color online) The dependence of the tip- and substrateadsorbate temperatures $T_{\mathrm{a}}$ and $T_{\mathrm{b}}$ on the tip-substrate separation $d$. For the same parameters as in Fig. 3 and $\tau_{\mathrm{b}}=3 \times 10^{-8} \mathrm{~s}$. 
relaxation time ${ }^{22} \tau_{\mathrm{b}}^{*} \approx 10^{-10} \mathrm{~s}$. We assume a Pt tip at room temperature with an adsorbed ${ }^{13} \mathrm{C}^{18} \mathrm{O}$ with $\omega_{\mathrm{a}} \approx 2064 \mathrm{~cm}^{-1}$ and $^{23} \tau_{\mathrm{a}}^{*} \approx 3 \times 10^{-12}$ s mainly due to decay by excitation of electron-hole pairs. Using the experimental measured vibrational polarizability $\alpha_{\mathrm{av}} \approx 0.2 \AA^{3}$ and $\alpha_{\mathrm{bv}} \approx 0.04 \AA^{3}$ and assuming a tip-substrate separation $d=1 \mathrm{~nm}$ we get $s \approx 20$ and $2\left(\omega_{\mathrm{a}}-\omega_{\mathrm{b}}\right) \tau_{\mathrm{a}}^{*} \approx 16$. Thus from (7) we get $r \approx 10^{6}$ and from (13) we get $T_{\mathrm{b}} \approx 300 \mathrm{~K}$ where we have assumed the tip temperature $T_{0}=300 \mathrm{~K}$. The $\mathrm{CO} / \mathrm{NaCl}$ case is an extreme case because of the exceptionally long vibrational energy relaxation time. However, the analysis presented above remains unchanged for any $\tau_{\mathrm{b}}$ larger than $10^{-8} \mathrm{~s}$, so the conclusions are very general. Thus we expect strong heating effects due to photon tunneling for high-frequency modes in adsorbed layers or films on insulating substrates.

The temperature increase for the $\mathrm{C}-\mathrm{O}$ stretch vibration found above is similar to the temperature increase observed (or calculated) for $\mathrm{CO}$ on $\operatorname{Pd}(110)$ during STM experiments. $^{20}$ In this the case the excitation of the C-O stretch vibration is caused by inelastic tunneling. The temperature increase in the $\mathrm{C}-\mathrm{O}$ stretch mode resulted in $\mathrm{CO}$ diffusion as a result of energy transfer to the parallel frustrated translation because of anharmonic coupling. This has been observed for CO molecules on Pd (110) (Ref. 20) and on Ag (110) (Ref. 24). We expect similar decay processes for vibrational excited $\mathrm{CO}$ on $\mathrm{NaCl}$ resulting, e.g., in diffusion or desorption of the $\mathrm{CO}$ molecule.

To summarize, we have calculated the temperature increase in adsorbed molecules due to photon tunneling from a tip. The temperature increase can be very large, in particular if the vibrational mode has a long vibrational lifetime. The temperature increase may induce diffusion, desorption, or other reactions, and we suggest that localized photon tunneling may be useful for local surface modification in adsorbate layers and thin adsorbed films. This technique is complementary to vibrational heating with STM, since STM can only be performed on conducting substrates, while the vibrational heating from photon tunneling is also possible for AFM and is particularly effective for insulating substrates because of the absence of electron-hole pair induced vibrational damping.

B.P. thanks the Japan Society for the Promotion of Science (JSPS) for support. H.U. was supported by a Grant-inAid for Scientific Research (B) from JSPS.
${ }^{1}$ D. Polder and M. Van Hove, Phys. Rev. B 4, 3303 (1971).

${ }^{2}$ M. L. Levin, V. G. Polevoy, and S. M. Rytov, Zh. Eksp. Teor. Fiz. 79, 2087 (1980) [Sov. Phys. JETP 52, 1054 (1980)].

${ }^{3}$ J. B. Pendry, J. Phys.: Condens. Matter 11, 6621 (1999).

${ }^{4}$ A. Majumdar, Annu. Rev. Mater. Sci. 29, 505 (1999).

${ }^{5}$ A. I. Volokitin and B. N. J. Persson, Phys. Rev. B 63, 205404 (2001); Phys. Low-Dimens. Semicond. Struct. 5/6, 151 (2001).

${ }^{6}$ A. I. Volokitin and B. N. J. Persson, Phys. Rev. B 65, 115419 (2002).

${ }^{7}$ J. P. Mulet, K. Joulin, R. Carminati, and J. J. Greffet, Appl. Phys. Lett. 78, 2931 (2001).

${ }^{8}$ A. I. Volokitin and B. N. J. Persson, JETP Lett. 78, 457 (2003).

${ }^{9}$ K. Joulain, J. P. Mulet, F. Marquier, R. Carminati, and J. J. Greffet, Surf. Sci. Rep. 57, 59 (2005).

${ }^{10}$ E. Riedo (private communication).

${ }^{11}$ K. Liu and S. Gao, Phys. Rev. Lett. 95, 226102 (2005).

${ }^{12}$ S. G. Tikhodeev and H. Ueba, Phys. Rev. B 70, 125414 (2004).

${ }^{13}$ B. N. J. Persson and M. Persson, Solid State Commun. 36, 175 (1980).

${ }^{14}$ M. Head-Gordon and J. C. Tully, Phys. Rev. B 46, 1853 (1992).

${ }^{15}$ B. N. J. Persson, Phys. Rev. B 44, 3277 (1991).

${ }^{16}$ H. C. Chang and G. E. Ewing, in Vibrations at Surfaces 1990, edited by Y. T. Chabal, F. M. Hoffmann, and G. P. Williams
(Elsevier, Amsterdam, 1990), p. 39.

${ }^{17}$ B. N. J. Persson, Sliding Friction, Physical Principles and Application, 2nd ed. (Springer, Heidelberg, 2000).

${ }^{18}$ A. I. Volokitin and B. N. J. Persson, in Inelastic Energy Transfer in Interactions with Surfaces and Adsorbates, edited by B. Gumhalter, A. C. Levi, and F. Flores (World Scientific, Singapore, 1993), pp. 217-248.

${ }^{19}$ Since the final states in energy relaxation and pure dephasing are different, the quantum mechanical interference between the two processes can be neglected and the total decay rate is the sum of the energy relaxation rate and the pure dephasing rate.

${ }^{20}$ T. Komeda, Y. Kim, M. Kawai, B. N. J. Persson, and H. Ueba, Science 295, 2055 (2002).

${ }^{21}$ J. I. Pascual, N. Lorente, Z. Song, H. Conrad, and H.-P. Rust, Nature (London) 432, 525 (2003).

${ }^{22}$ H. C. Chang, H. H. Richardson, and G. E. Ewing, J. Chem. Phys. 89, 7561 (1988).

${ }^{23}$ J. D. Beckerle, M. P. Casassa, E. J. Heilweil, R. R. Cavanagh, and J. C. Stephenson, in Vibrations at Surfaces 1990, edited by Y. T. Chabal, F. M. Hoffmann, and G. P. Williams (Elsevier, Amsterdam, 1990), p. 17.

${ }^{24}$ J. R. Hahn and W. Ho, Phys. Rev. Lett. 87, 166102 (2001). 